Tomonori Kawasaki $^{1}$, Kyoichi Kaira ${ }^{2}$, Yasuhiro Nakamura ${ }^{3}$, Hisao Imai ${ }^{2}$, Bierta Barfod ${ }^{1}$

${ }^{1}$ Department of Pathology, Saitama Medical University International Medical Center, Hidaka, Japan; ${ }^{2}$ Department of Respiratory Medicine, Saitama Medical University International Medical Center, Hidaka, Japan; ${ }^{3}$ Department of Skin Oncology, Saitama Medical University International Medical Center, Hidaka, Japan

Contact: Dr Tomonori Kawasaki.

E-mail: tomo.kawasaki.14@gmail.com

1. Razvi H, Tsang JY, Poon IK, et al. INSM1 is a novel prognostic neuroendocrine marker for luminal B breast cancer. Pathology 2021; 53: $170-8$

2. Goto Y, De Silva MG, Toscani A, et al. A novel human insulinomaassociated cDNA, IA-1, encodes a protein with "zinc-finger" DNAbinding motifs. J Biol Chem 1992; 267: 15252-7.

3. Kawasaki T, Kaira K. Insulinoma-associated protein 1 (INSM1) expression in breast carcinomas with neuroendocrine morphologies: application and future prospective. Virchows Arch 2020; Oct 6: https://doi.org/ 10.1007/s00428-020-02935-0.

4. Kawasaki T, Inoue A, Mochizuki K, et al. Neuroendocrine ductal carcinoma in situ, comedo type, of the breast detected by screening mammography: a potentially pre-invasive counterpart of high grade neuroendocrine tumours. Pathology 2012; 44: 273-5.

5. Kawasaki T, Bussolati G, Castellano I, et al. Small-cell carcinoma of the breast with squamous differentiation. Histopathology 2013; 63: 739-41

6. Kawasaki T, Kondo T, Nakazawa T, et al. Is CD56 a specific and reliable neuroendocrine marker for discriminating between endocrine/neuroendocrine ductal carcinoma in situ and intraductal papilloma of the breast? Pathol Int 2011; 61: 49-51.

DOI: https://doi.org/10.1016/j.pathol.2020.12.001

\section{Re: INSM1 is a novel prognostic neuroendocrine marker for luminal B breast cancer: author reply}

Sir,

This letter is in response to the questions raised by Kawasaki et al. regarding our recently published paper on INSM1 as a novel prognostic neuroendocrine (NE) marker in breast cancer. We appreciate their comments on our study and thank them for sharing their experience. ${ }^{2}$ One question that they raised was about the proportion and morphology of breast cancers showing INSM1+/chromogranin (CG)-/synaptophysin (SYN)-. The other point was on the morphological findings of invasive cancers showing CD56 reactivity exclusively.

In our INSM1 study, in total there were 1375 cases with complete staining results on INSM1, CG and SYN. Among them, $1159(84.3 \%)$ cases expressed neither markers, 72 $(5.2 \%)$ cases showed co-expression of INSM1 with either CG and/or SYN, and $121(8.8 \%)$ cases expressed only CG and/or SYN with no INSM1. There were $23(1.7 \%)$ cases showing INSM1 expression exclusively (CG-/SYN/INSM1+, INSM only positive). In our report, the INSM1+ cases with NE morphology in the illustration also expressed CG and SYN, thus were classical neuroendocrine tumors (NET). For the 23 INSM1 only positive cases, the majority belonged to the luminal subtypes (11 luminal $\mathrm{A}$ and 10 luminal B cases; 91.3\%). A high proportion of them were of low grade and invasive breast carcinoma of no special type (IBC-NST). Despite being mostly IBC-NST, NE morphology (clear cell, spindle and carcinoid morphology) was observed in four INSM1 positive only cases. Two of these were low grade with predominant NE morphology which were categorised as NET. The other two showed 50-70\% NE morphology and were classified as mixed IBC-NST with NET. Details of the INSM1 only positive cases are shown in Table 1. We agreed with Kawasaki et al. as reported in their recent study $^{3}$ that INSM1 expression indeed highlighted additional NE cases. Given its similar clinicopathological association as CG and SYN, it could be a potential useful diagnostic marker for NE features in breast cancer.

Regarding CD56, 54 (4.0\%) of 1351 cases showed exclusive expression of this marker. Among them, NE morphology was found in eight cases. Of these, five cases showed predominant NE morphology and were classified as NET; the remaining three cases were mixed IBC-NST with NET. However, a lower proportion of them (35 cases, 64.8\%) were of luminal subtypes. Although CD56 could also

Table 1 Detailed features on cases showing INSM1+/SYN-/CG- $(n=23)$

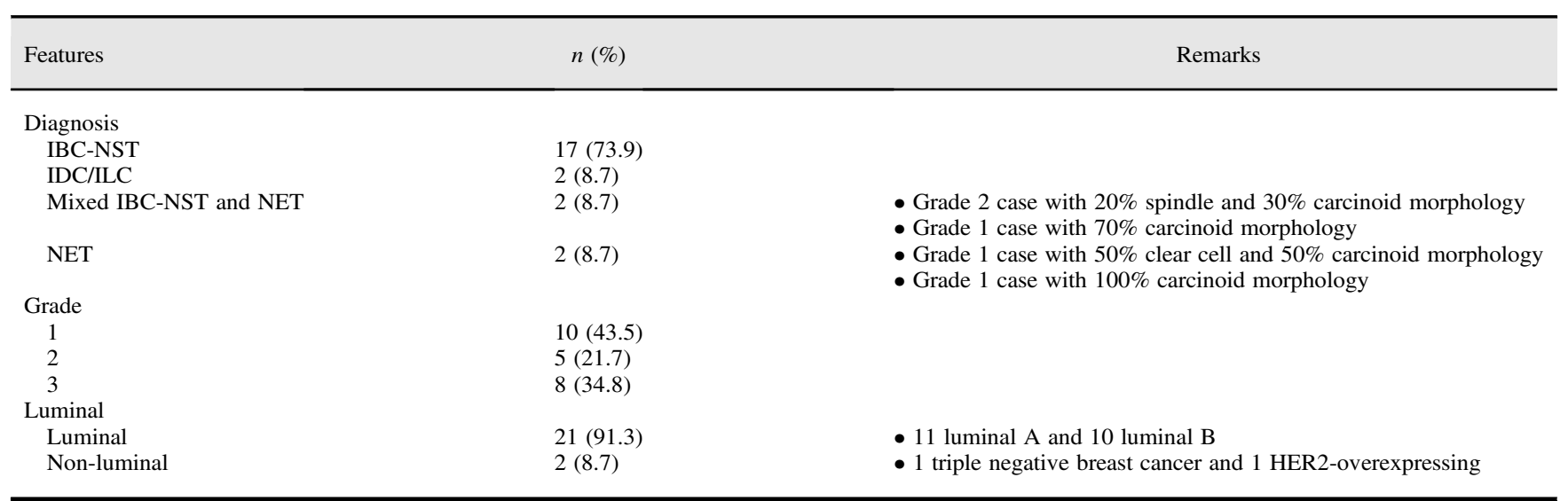

IBC-NST, invasive breast carcinoma of no special type; IDC, invasive ductal carcinoma; ILC, invasive lobular carcinoma. 
highlight additional NE cases, these CD56 only positive cases appeared to have distinct features from typical NE cases. We have recently compared the clinicopathological features and outcome of breast cancer based on the level of CD56, CG and SYN. ${ }^{4}$ CD56 showed a positive correlation with CK14 but not CG and SYN. Further comparison was carried out between cases with SYN/CG+CD56+/- and $\mathrm{CG}-/ \mathrm{SYN}-/ \mathrm{CD} 56+$ (CD56 only positive) cases. We found that CD56 only positive cases did not show typical NE associations; conversely, they were associated with younger patient ages, non-luminal subtypes and high levels of expression of basal markers (EGFR, p63, CK5/6 and CK14). In addition, CD56 only positive cases showed more similar disease free survival to IBC without any NE features, than cases with SYN/CG+ CD56+/- expression. It appeared that CD56 only positive cases behaved more like IBC without any $\mathrm{NE}$ features, differing from the typical NE cases. Further studies are required to confirm the relevance of CD56 as a NE marker in breast cancer.

NE differentiation in breast cancer has been underdiagnosed in routine clinical settings. Apart from not being routinely tested, some cases can still be missed by the common NE markers, namely SYN and CG. There is an unmet need for $\mathrm{NE}$ marker development in breast cancers. On the basis of current data, as a NE marker, INSM1 appears to hold better promise than CD56.
Conflicts of interest and sources of funding: The authors state that there are no conflicts of interest to disclose.

\section{Huda Razvi, Julia Y. Tsang, Gary M. Tse}

Department of Anatomical and Cellular Pathology, Prince of Wales Hospital, The Chinese University of Hong Kong, Shatin, NT, Hong Kong

Contact Dr Gary M. Tse.

E-mail: garytse@cuhk.edu.hk

1. Razvi H, Tsang JY, Poon IK, et al. INSM1 is a novel prognostic neuroendocrine marker for luminal B breast cancer. Pathology 2021; 53: $170-8$

2. Kawasaki T, Kaira K, Nakamura Y, Imai H, Barfod B. Re: INSM1 is a novel prognostic neuroendocrine marker for luminal B breast cancer. Pathology 2020; 53: 292-3.

3. Kawasaki T, Kaira K. Insulinoma-associated protein 1 (INSM1) expression in breast carcinomas with neuroendocrine morphologies: application and future prospective. Virchows Arch 2020; Oct 6: https://doi.org/ 10.1007/s00428-020-02935-0.

4. Lai BS, Tsang JY, Poon IK, et al. The clinical significance of neuroendocrine features in invasive breast carcinomas. Oncologist 2020; 25 e1318-29.

DOI: https://doi.org/10.1016/j.pathol.2020.12.002 